\title{
Optimal local discrimination of two multipartite pure states
}

\author{
S. Virmani ${ }^{1}$, M. F. Sacchi ${ }^{1,2}$, M. B. Plenio ${ }^{1}$ and D. Markham ${ }^{1}$ \\ ${ }^{1}$ Optics Section, Blackett Laboratory, Imperial College, London SW7 $2 B W$, \\ United Kingdom. \\ 2 Dipartimento di Fisica 'A. Volta', Università di Pavia and Unità INFM, via A. \\ Bassi 6, I27100 Pavia, Italy.
}

PACS numbers: 03.67.-a, 3.67.Lk

\begin{abstract}
In a recent paper, Walgate et. al. [1] demonstrated that any two orthogonal multipartite pure states can be optimally distinguished using only local operations. We utilise their result to show that this is true for any two multiparty pure states, in the sense of inconclusive discrimination. There are also certain regimes of conclusive discrimination for which the same also applies, although we can only conjecture that the result is true for all conclusive regimes. We also discuss a class of states that can be distinguished locally according to any discrimination measure, as they can be locally recreated in the possession of one party. A consequence of this is that any two maximally entangled states can always be optimally discriminated locally, according to any figure of merit.
\end{abstract}

\section{Introduction}

Entanglement lies at the heart of many aspects of quantum information theory and it is therefore desirable to understand its structure as well as possible. One attempt to improve our understanding of entanglement is the study of our ability to perform information theoretic tasks locally on non-local states, such as the local implementation of non-local quantum gates [2], telecloning [3], the remote manipulation and preparation of quantum states [4] or the recently studied question of the local discrimination of non-local states by a variety of authors. In [1] it was shown that any two orthogonal pure states can be perfectly discriminated locally, whereas in [5] examples of two orthogonal mixed states were presented which cannot be distinguished perfectly locally. Another surprising development is that there exist bases of product orthogonal pure states which cannot be locally reliably discriminated, despite the fact that

Preprint submitted to Elsevier Preprint 22 October 2018 
each state in the basis contains no entanglement [6]. Here we discuss the issue of discriminating two non-orthogonal pure states locally, and show that in this regime the optimal global procedure can be achieved.

\section{Background Material}

We will begin by recalling the optimal method for discriminating two states when we are not restricted to local actions. Suppose that we are given one of two mixed states $\rho_{1}$ and $\rho_{2}$ (not necessarily orthogonal) with prior probabilities $p_{1}$ and $p_{2}$ respectively, and we have been asked to optimally determine which state we have actually been given. Helstrom [7] (see also [8] for a very readable account) showed that the optimal discrimination protocol is as follows. Consider the following Hermitian matrix:

$$
\Delta=p_{1} \rho_{1}-p_{2} \rho_{2}
$$

As it is Hermitian, $\Delta$ has a basis of orthogonal eigenvectors $\left\{\left|\delta_{i}\right\rangle\right\}$. In general $k$ of these eigenstates will have positive (or zero) eigenvalues and $n-k$ will be negative, where $n$ is the dimension of $\Delta$. We will denote the positive eigenstates as $\left\{\left|\delta_{i}^{+}\right\rangle\right\}, i=1 \ldots k$ and the negative eigenstates as $\left\{\left|\delta_{i}^{-}\right\rangle\right\}, i=k+1 \ldots n$. The optimal measurement is then to measure in this basis and whenever one of the positive eigenstates is obtained return the answer $\rho_{1}$ and whenever a negative eigenstate is obtained return the answer $\rho_{2}$. In this case the probability of error will be given by:

$$
P_{E}=\sum_{i=1}^{k} p_{2} \operatorname{tr}\left(\rho_{2}\left|\delta_{i}^{+}\right\rangle\left\langle\delta_{i}^{+}\right|\right)+\sum_{i=k+1}^{n} p_{1} \operatorname{tr}\left(\rho_{1}\left|\delta_{i}^{-}\right\rangle\left\langle\delta_{i}^{-}\right|\right)
$$

This is the optimal probability which can be achieved by any POVM [7]. In particular, in the case of two pure states $\left|\psi_{1}\right\rangle,\left|\psi_{2}\right\rangle$ with prior probabilities $p_{1}$ and $p_{2}$ respectively, the optimal measurement consists of measuring in the basis $\{|+\rangle,|-\rangle\}$, where $\{|+\rangle,|-\rangle\}$ are the positive and negative eigenvalue eigenstates of the matrix $\Delta=p_{1}\left|\psi_{1}\right\rangle\left\langle\psi_{1}\left|-p_{2}\right| \psi_{2}\right\rangle\left\langle\psi_{2}\right|$. Although in this case $\Delta$ will in general also have many eigenstates of eigenvalue zero, they are not important as they will never occur in any measurement of the two pure states. The probability of error is then given by:

$$
P_{E}=p_{2} \operatorname{tr}\left(\left|\psi_{2}\right\rangle\left\langle\psi_{2} \mid+\right\rangle\langle+|\right)+p_{1} \operatorname{tr}\left(\left|\psi_{1}\right\rangle\left\langle\psi_{1} \mid-\right\rangle\langle-|\right)
$$

It will be useful to note that the states $\{|+\rangle,|-\rangle\}$ are constructed in such a way that we can always write: 


$$
\begin{aligned}
& \left|\psi_{1}\right\rangle=a|+\rangle+b|-\rangle \\
& \left|\psi_{2}\right\rangle=c|+\rangle+d|-\rangle
\end{aligned}
$$

for some complex coefficients $a, b, c, d$.

We will later require the results of Walgate et. al. [1] to show that this probability of error can be achieved using a locally implementable measurement. They showed that any two bipartite orthogonal pure states $|+\rangle$ and $|-\rangle$ on Hilbert space $H_{A} \otimes H_{B}$ can be expressed in the following way:

$$
\begin{aligned}
|+\rangle & =\sum_{i} \alpha_{i}|i\rangle\left|\eta_{i}\right\rangle \\
|-\rangle & =\sum_{i} \beta_{i}|i\rangle\left|\mu_{i}\right\rangle
\end{aligned}
$$

where the $\{|i\rangle\}$ form an orthonormal basis for $H_{A}$ and the $\left|\eta_{i}\right\rangle,\left|\mu_{i}\right\rangle$ are normalised states satisfying:

$$
\left\langle\mu_{i} \mid \eta_{i}\right\rangle=0 .
$$

Other than this condition the $\left|\eta_{i}\right\rangle$ and $\left|\mu_{i}\right\rangle$ needn't be orthogonal, although we will treat them as normalised (this is slightly different to the convention used in [1]). By proving the existence of such a decomposition, [1] showed how two orthogonal pure states can be discriminated locally. First Alice must measure her side in the basis $\{|i\rangle\}$. If she obtains the outcome $|j\rangle$, Bob must then measure in an orthonormal basis containing the states $\left|\eta_{j}\right\rangle$ and $\left|\mu_{j}\right\rangle$. As $\left\langle\mu_{i} \mid \eta_{i}\right\rangle=0 \forall i$, this is always possible. If the outcome corresponds to $\left|\eta_{j}\right\rangle$ then the state was $|+\rangle$, if the outcome corresponds to $\left|\mu_{j}\right\rangle$ then the state was $|-\rangle$. In more formal terms this corresponds to performing a locally-implementable measurement with elements $\left\{\left\{\left|i \eta_{i}\right\rangle\left\langle i \eta_{i}\right|\right\},\left\{\left|i \mu_{i}\right\rangle\left\langle i \mu_{i}\right|\right\}\right\}$, such that if the outcomes $\left\{\left|i \eta_{i}\right\rangle\left\langle i \eta_{i}\right|\right\}$ are obtained we know that the state was $|+\rangle$ and if the outcomes $\left\{\left|i \mu_{i}\right\rangle\left\langle i \mu_{i}\right|\right\}$ are obtained then the state was $|-\rangle$.

\section{Optimal local inconclusive discrimination of two multipartite pure states}

They key point in generalising this result to non-orthogonal states is that in order to discriminate two nonorthogonal pure states $\left|\psi_{1}\right\rangle$ and $\left|\psi_{2}\right\rangle$ optimally we need to measure in a basis of the two orthogonal states $\{|+\rangle,|-\rangle\}$ given by the eigenvectors of $\Delta$. So we can 'pretend' that we are essentially trying to discriminate between one of the two orthogonal states $\{|+\rangle,|-\rangle\}$, and implement the local measurment $\left\{\left\{\left|i \eta_{i}\right\rangle\left\langle i \eta_{i}\right|\right\},\left\{\left|i \mu_{i}\right\rangle\left\langle i \mu_{i}\right|\right\}\right\}$. If the outcomes 
$\left\{\left|i \eta_{i}\right\rangle\left\langle i \eta_{i}\right|\right\}$ are obtained we say that the original state was $\left|\psi_{1}\right\rangle$, whereas if the outcomes $\left\{\left|i \mu_{i}\right\rangle\left\langle i \mu_{i}\right|\right\}$ are obtained we say that the original state was $\left|\psi_{2}\right\rangle$. Indeed, with this POVM the probability of error will be given by:

$$
P_{E}=\sum_{i} p_{2} \operatorname{tr}\left(\left|\psi_{2}\right\rangle\left\langle\psi_{2} \mid i \eta_{i}\right\rangle\left\langle i \eta_{i}\right|\right)+p_{1} \operatorname{tr}\left(\left|\psi_{1}\right\rangle\left\langle\psi_{1} \mid i \mu_{i}\right\rangle\left\langle i \mu_{i}\right|\right)
$$

It is easy to verify (using equation (4)) that this probability of error is equal to that of equation (2), thus demonstrating that we can achieve the optimal discrimination probability for two bipartite pure states by using only local operations.

The generalisation to multipartite pure states follows in a similar manner. Walgate et. al. [1] pointed out that the decomposition (5) also implies that any two multipartite orthogonal states can be discriminated using only local actions. Imagine two orthogonal three party pure states shared between Alice, Bob and Chris. Bob and Chris can at first be grouped as one party, and then the decomposition (5) can be applied to the Alice-(Bob,Chris) split. Then Alice measures her side according to the above bipartite protocol, leaving (Bob-Chris) in one of two orthogonal states depending upon which tripartite pure state they all originally shared. Then Bob \& Chris can simply apply the bipartite protocol to tell which state they had. The same method can easily be seen to generalize to any two multipartite orthogonal pure states. This result in turn can be used to show, following exactly the same argument as the above bipartite one, that any two multiparty pure states can be distinguished in an optimal manner using local operations only.

It is worth pointing out that the above argument also extends to some mixed state scenarios. In particular, when the two mixed states to be distinguished collectively span only a two dimensional space then there will be only two non-trivial eigenstates of the matrix $\Delta$. In this situation the above reasoning implies that two mixed states with this property can be optimally distinguished locally.

\section{Conclusive discrimination}

The above arguments apply to the case of inconclusive discrimination, in which we have to say that the state we are given is either $\rho_{1}$ or $\rho_{2}$, but we are allowed to sometimes make errors, the probability of which me must minimise. However, there is also the case of conclusive discrimination, in which we are allowed to give three outcomes; $\rho_{1}, \rho_{2}$ or 'don't know'. In this case, we must never be wrong when we return outcomes $\rho_{1}$ or $\rho_{2}$, and we have to try to minimise the probability of getting the 'don't know' outcome. It is therefore also of 
interest to know how to optimise conclusive local discrimination. The optimal conclusive protocol for globally discriminating two pure states is discussed in references $[9,10]$. In some situations this protocol reduces to performing an orthogonal measurement of two orthogonal states, in which case exactly the same arguments as above apply again, and the optimal discrimination protocol can be matched by a local one. In particular, this applies to situations in which [10] (taking $p_{2} \geq p_{1}$ ):

$$
\left|\left\langle\psi_{1} \mid \psi_{2}\right\rangle\right| \geq\left(\frac{p_{1}}{p_{2}}\right)^{1 / 2}
$$

In which case the optimal discrimination probability is given by:

$$
P_{\text {success }}=p_{1}\left(1-\left|\left\langle\psi_{1} \mid \psi_{2}\right\rangle\right|^{2}\right) \text {. }
$$

However, the general optimal conclusive case requires three outcomes to have non-zero probability (corresponding to $\rho_{1}, \rho_{2}$ and 'don't know'), thus requiring a minimum of three orthogonal pure states to be distinguished locally. As the question of locally discriminating three orthogonal pure states is currently unanswered, we were not able to extend these results to the entire conclusive case. However, we were able to make some progress in some situations with equal prior probabilities, $p_{1}=p_{2}$. In this situation, [9] show that the optimal global discrimination probability for two pure states is given by:

$$
1-P_{E}=1-\left|\left\langle\psi_{1} \mid \psi_{2}\right\rangle\right|
$$

It is easy to verify that in the case of two product pure states if Alice and Bob implement such a measurement independently, and then compare their results, then the probability that they both fail is equal to the minimal global failure probability. Hence two product pure states of prior probability $1 / 2$ can be locally discriminated optimally. It has also recently been shown that any two product pure states with arbritrary prior probabilities can be optimally conclusively discriminated locally [11]. For more general two qubit pure states, the situation is not so straightforward. We considered trying to perform local one-dimensional projections on Alice side which would give her no information, and leave Bob's particle in residual states which could perhaps be easily distinguished from one another. Although this is a very restricted protocol, it is more suitable for analysis than a more general scheme. We will write the two pure states to be distinguished in terms of two matrices $\Psi_{1}$ and $\Psi_{2}$ in the following manner (see, for example [12]):

$$
\left|\psi_{1}\right\rangle=\sum_{i, j}\left(\Psi_{1}\right)_{i j}|i\rangle|j\rangle
$$




$$
\left|\psi_{2}\right\rangle=\sum_{i, j}\left(\Psi_{2}\right)_{i j}|i\rangle|j\rangle .
$$

In this notation, if Alice performs a local measurement an orthonormal basis consisting of the states $|\chi\rangle$ and $\left|\chi^{\perp}\right\rangle$, then the condition

$$
\left\langle\chi\left|\Psi_{1} \Psi_{1}^{\dagger}\right| \chi\right\rangle=\left\langle\chi\left|\Psi_{2} \Psi_{2}^{\dagger}\right| \chi\right\rangle
$$

guarantees that the states after Alice's measurement on Bob's side are given with the same probability as the initial two pure states $\left|\psi_{1}\right\rangle$ or $\left|\psi_{2}\right\rangle$. If Bob then were to apply the optimal conclusive discrimination on his side, the overall probability of successful discrimination would be given by

$$
\tilde{P}_{D}=1-\left|\left\langle\chi\left|\Psi_{2} \Psi_{1}^{\dagger}\right| \chi\right\rangle\right|-\left|\left\langle\chi^{\perp}\left|\Psi_{2} \Psi_{1}^{\dagger}\right| \chi^{\perp}\right\rangle\right|,
$$

whereas the global optimal probability is equal to

$$
\begin{aligned}
P_{D} & =1-\left|\left\langle\chi\left|\Psi_{2} \Psi_{1}^{\dagger}\right| \chi\right\rangle+\left\langle\chi^{\perp}\left|\Psi_{2} \Psi_{1}^{\dagger}\right| \chi^{\perp}\right\rangle\right| \\
& =1-\operatorname{Trace}\left[\Psi_{2} \Psi_{1}^{\dagger}\right]=1-\left|\left\langle\psi_{1} \mid \psi_{2}\right\rangle\right| .
\end{aligned}
$$

If both equation (12) and $\tilde{P}_{D}=P_{D}$ are satisfied then we can say that such a protocol is optimal. We have numerically and analytically confirmed that this is the case for a large set of states, although we were not able to prove that such a solution is always possible. In cases with unequal prior probability this method does not always work optimally, although there may still be room to improve the protocol by dropping the condition that Alice learns nothing from her measurement. However, in such situations a much more intractable system of equations and inequalities has to be satisfied (see [10]), and we were not able to obtain any significant solutions.

\section{Local discrimination according to any figure of merit}

Given that we have considered the problem of optimal distinguishability under two quality measures, one could ask the question as to whether there are states that can be distinguished locally as well as globally according to any discrimination figure of merit. An interesting example of states for which this is possible is the set of Schmidt correlated states [13,14]. The method for distinguishing these states is in fact very powerful, as it leads to the solution of a more general problem. The reason for this is that we can transform these states locally into local states with the same distinguishability properties. Consider the special case of two Schmidt correlated pure states, say: 


$$
\begin{aligned}
& \phi_{1}=\alpha|00\rangle+\beta|11\rangle \\
& \phi_{2}=\gamma|00\rangle+\delta|11\rangle .
\end{aligned}
$$

Imagine that we have been given one of these states with some arbitrary prior probabilities. If Alice performs a measurement in the basis $\{|0\rangle+|1\rangle,|0\rangle-|1\rangle\}$, then she will leave Bob's particle in some residual state. It is easy to check that if Alice informs Bob of the measurement outcome, then he can easily rotate his state back to either $\alpha|0\rangle+\beta|1\rangle$ or $\gamma|0\rangle+\delta|1\rangle$, depending upon whether $\phi_{1}$ or $\phi_{2}$ respectively was originally shared (this solution can also be found according to the discussion in Eqs. (12-14)). Bob can then locally recreate the state that he and Alice shared by adding an ancilla qubit in the state $|0\rangle$ and performing a control-NOT operation. Surprisingly, this means that for Schmidt correlated states the optimal global discrimination protocol can be achieved locally, depsite the fact that the reduced density matrices are so similar. This method can easily be generalised to any number of Schmidt correlated mixed states of any dimension [15] and any number of particles (where for more than two particles Schmidt correlated would refer to mixtures of cat-states with the same cat-state basis).

The question then arises, when is it possible to express two pure states in a Schmidt correlated form? In the following section we discuss necessary and sufficient conditions for this to be possible. These conditions are satisfied by many states, particularly by those with sufficient degeneracy amongst their Schmidt coefficients. An interesting consequence is that any two maximally entangled states can always be expressed in Schmidt correlated form, thus showing that two maximally entangled states can always be discriminated locally optimally, regardless of which figures of merit are chosen.

\section{When two pure states can be written in Schmidt Correlated form}

In this section we will discuss when it is possible to express two general pure states in Schmidt correlated form. Using the matrix notation of equation (11), it can be shown that a local basis change corresponding to unitaries $U \otimes V$ transforms the matrices representing the two pure states $\left|\psi_{1}\right\rangle$ and $\left|\psi_{2}\right\rangle$ as $U \Psi_{1} V^{T}$ and $U \Psi_{2} V^{T}$ respectively. Two states can be brought into maximally correlated form by such local unitaries if both the resulting state matrices are diagonal, i.e.:

$$
\begin{aligned}
& \left(U \Psi_{1} V^{T}\right)_{i j}=x_{i} \delta_{i j} \\
& \left(U \Psi_{2} V^{T}\right)_{i j}=y_{i} \delta_{i j}
\end{aligned}
$$


for some complex $\left\{x_{i}\right\}$ and $\left\{y_{i}\right\}$. It is a well known consequence of the singular value decomposition theorem [16] of matrix analysis that any square matrix $\Psi$ can be brought into diagonal form by two unitaries $U, V$ acting as $U \Psi V^{T}$. Our question now concerns a modification of this result - when can two matrices $\Psi_{1}$ and $\Psi_{2}$ be brought into diagonal form by the same unitaries $U$ and $V$ ? In cases where the Schmidt decomposition of each pure state is unique, it may be easy to see if the two pure states are already Schmidt correlated. However, in cases where there is degeneracy amongst the Schmidt coefficients it is not always apparent whether some local basis change would bring both states into Schmidt correlated form. A necessary and sufficient condition is presented in [17]:

Lemma 1: Two square matrices $F$ and $G$ can be brought into diagonal form by the same unitaries $U, V$ iff both $F G^{\dagger}$ and $G^{\dagger} F$ are normal, i.e. they commute with their Hermitian conjugates.

In more physical terms, this means that two pure states can be brought into Schmidt correlated form by the same local unitaries iff the corresponding matrix representations $F$ and $G$ satisfy:

$$
\left[F G^{\dagger}, G F^{\dagger}\right]=0
$$

and

$$
\left[G^{\dagger} F, F^{\dagger} G\right]=0
$$

Although these conditions at first appear quite opaque, there are some cases where they give valuable insights. As mentioned earlier, an immediate consequence is that two maximally entangled states can always be brought into Schmidt correlated form. This surprising result follows from the fact that the reduced density matrices for a pure state represented by a matrix $F$ are given by $\rho_{A}(F)=F F^{\dagger}$ and $\rho_{B}^{T}(F)=F^{\dagger} F$. As maximally entangled states have reduced density matrices proportional to the identity, we can immediately see that any $F, G$ corresponding to maximally entangled states are proportional to unitary matrices. It is straightforward to verify that any such matrices satisfy the conditions of Lemma 1. In fact, we can easily go further and explicitly construct the local unitaries which will bring any two maximally entangled states into Schmidt correlated form. Without loss of generality, we can set the local bases such that $F$ is real and proportional to the identity (i.e. $F_{i j}=1 / \sqrt{d} \delta_{i j}$ ). Hence we can consider $F$ to be invariant under any unitary matrix $U$ acting as $U F U^{\dagger}$. As $G$ is unitary, it can be diagonalised by a unitary transformation $\sigma G \sigma^{\dagger}$, where $\sigma$ is another unitary matrix. This corresponds to the physical operation $\sigma \otimes \sigma^{*}$. Hence $G$ will be made diagonal by such a transformation, and as $F$ is invariant under this operation, we will have succeeded in bringing 
both $F$ and $G$ into Schmidt correlated form.

It is in fact the degeneracy of the Schmidt coefficients of maximally entangled states which allows them to be brought into Schmidt correlated form in this way. In the rest of this section we will sketch the proof of Lemma 1, thereby seeing why this is the case. Without loss of generality we can set $F$ to be a diagonal positive semidefinite matrix with degenerate eigenvalues $\alpha_{i}$, each of degeneracy $d_{i}$. In particular let us partition $F$ into blocks determined by the degeneracies in its eigenvalues, i.e.

$$
F=\left(\begin{array}{ccccc}
F_{1} & 0 & 0 & 0 & 0 \\
0 & F_{2} & 0 & 0 & 0 \\
0 & 0 & . & . & . \\
0 & 0 & . & . \\
0 & 0 & . & & .
\end{array}\right),
$$

where the $F_{i}=\alpha_{i} I_{d_{i}}$, and $I_{d_{i}}$ is a $d_{i} \times d_{i}$ identity matrix.

In [18] it has been shown that if $F$ is of this form and both $F G^{\dagger}$ and $G^{\dagger} F$ are normal, then $G$ must have the same block structure as $F$, i.e.

$$
G=\left(\begin{array}{ccccc}
G_{1} & 0 & 0 & 0 & 0 \\
0 & G_{2} & 0 & 0 & 0 \\
0 & 0 & . & . & . \\
0 & 0 & . & . \\
0 & 0 & . & .
\end{array}\right),
$$

and in addition we must have that each $G_{i}$ is a normal matrix of dimension $d_{i} \times$ $d_{i}$. As each $G_{i}$ is normal, it can be diagonalised by a unitary transformation, say $U_{i}$. Hence the unitary matrix $W=\bigoplus_{i} U_{i}$ will diagonalise $G$ and leave $F$ unchanged (and hence diagonal). This corresponds to the physical operation $W \otimes W^{*}$. Hence any two matrices $F, G$ with the property that $F G^{\dagger}$ and $G^{\dagger} F$ are normal can be made Schmidt-correlated by local unitaries. The converse follows from performing a few lines of algebra to show that if $U F V^{T}=D_{1}$ and $U G V^{T}=D_{2}$, where $D_{1}$ and $D_{2}$ are diagonal, then both $F G^{\dagger}$ and $G^{\dagger} F$ must be normal.

If there is no degeneracy amongst the Schmidt coefficients of $F$, then $G$ will be forced to have a diagonal block structure. Hence if $F$ has no degeneracy, $G$ must be diagonal if it is possible to express the states in Schmidt correlated 
form, and the states will be 'obviously' Schmidt correlated from their Schmidt decomposition. If two pure states are not 'obviously' Schmidt correlated in this way, it will only be possible to use local unitaries to make them so if there is sufficient degeneracy in the Schmidt coefficients of both states.

\section{Conclusion}

We have demonstrated that any two multipartite pure states can be inconclusively discriminated optimally using only local operations. We have also shown that this is possible for certain mixed states and certain regimes of conclusive discrimination. We then turned our attention to finding sets of entangled states that can be recreated locally, thus allowing any global discrimination figure of merit to be achieved locally. We find that this is true for the Schmidt correlated states, and as a consequence this is also for any two maximally entangled states.

It would be interesting to know if there are many other states which can be locally recreated using other techniques. If this can be shown to apply to any two pure states, then we would know that two pure states can be distinguished optimally under any figure of merit using only local operations.

Acknowledgements. We would like to thank Jon Walgate and Vlatko Vedral for some illuminating discussions. We would also like to thank Koenraad Audenaart for drawing the results of [17] to our attention. SV would also like to thank Sougato Bose for interesting conversations on this issue in the past. This work was supported by the Leverhulme Trust, the European Union EQUIP IST-FET project, the UK Engineering and Physical Sciences Research Council, the European Science Foundation QIT programme and the Italian Ministero dell'Università e della Ricerca Scientifica e Tecnologica.

\section{References}

[1] J. Walgate, A. Short, L. Hardy and V. Vedral, Phys. Rev. Lett.85, 4972 (2000)

[2] J. Eisert, K.A. Jacobs, P. Papadopoulos and M.B. Plenio, Phys. Rev. A 62 (2000) 052317; D. Collins, N. Linden and S. Popescu, lanl e-print quantph/0005102; D. Gottesman, lanl e-print quant-ph/9903099.

[3] M. Murao, D. Jonathan, M.B. Plenio, and V. Vedral, Phys. Rev. A 59, 156 (1999). 
[4] H.-K. Lo, Phys. Rev. A 62, 012313 (2000); S.F. Huelga, J.A. Vaccaro, A. Chefles, and M.B. Plenio, Phys. Rev. A 63, 042303 (2001); C.H. Bennett et al, lanl e-print quant-ph/0006044; A. Pati, Phys. Rev. A 63, 014302 (2001).

[5] B. Terhal, D.P. DiVincenzo, D. Leung, quant-ph/0011042

[6] C.H. Bennett, D.P. DiVincenzo, C.A. Fuchs, T. Mor, E. Rains, P.W. Shor, J.A. Smolin, W.K. Wootters, Phys. Rev. A. 59, 1070 (1999).

[7] C. W. Helstrom, Quantum Detection and Estimation Theory, Academic Press, New York, 1976.

[8] C.A. Fuchs, PhD Thesis University of New Mexico also available as lanl e-print quant-ph/9601020.

[9] I. D. Ivanovic, Phys. Lett. A 123, 257 (1987); D. Dieks, Phys. Lett. A 126, 303 (1988); A. Peres, Phys. Lett. A 128, 19 (1988).

[10] G. Jaeger and A. Shimony, Phys. Lett. A 197, 83 (1995).

[11] Y-X Chen and D. Yang, quant-ph/0103111

[12] G. M. D'Ariano, P. Lo Presti and M. F. Sacchi, Phys. Lett. A. 272, 32 (2000)

[13] E. Rains, Phys. Rev. A 60, 179 (1999); Phys. Rev. A 63, 019902 (2001).

[14] The term Schmidt correlated [13] refers to any density matrix or set of density matrices which have eigenvectors possessing the same Schmidt basis. As an example, on $H_{2} \otimes H_{2}$ any density matrices which are mixtures of pure states of the form $\alpha|00\rangle+\beta|11\rangle$ (with the Schmidt basis fixed) fall into this class. Although [13] referred to such states as Maximally correlated, we will continue to use the term Schmidt correlated

[15] If Alice and Bob possess $d$-level particles each, with each Schmidt correlated state having Schmidt basis states $\left\{|k\rangle^{A}|k\rangle^{B}\right\}$, then the generalisation of this protocol is for Alice to measure in a basis of states $\{|\chi\rangle\}$, with each $|\chi\rangle$ having the property that $|\chi\rangle=1 / \sqrt{d} \Sigma_{k} \exp \left(i \theta_{k}\right)|k\rangle$. Such bases exist, so this protocol is possible.

[16] R. Bhatia, Matrix Analysis (Springer Graduate Texts in Mathematics vol. 169)

[17] C. Eckart and G. Young, Bull. Amer. Math. Soc. 45 (1939) 118-121. For a modern discussion see R. A. Horn and C. A Johnson, Matrix Analysis, CUP, p.426.

[18] N. A. Weigmann, J. Australian Math. Soc. 11 (1970) 329-339 